\title{
Analyzing Coordination of Upper and Lower Extremities in Human Gait
}

\author{
Duk-Jin Kim \\ University of Texas at Dallas \\ P.O. Box 75083 \\ Richardson, Texas 75083 \\ duk-jin.kim@utdallas.edu
}

\author{
Gaurav Pradhan \\ University of Texas at Dallas \\ P.O. Box 75083 \\ Richardson, Texas 75083 \\ gaurav@utdallas.edu
}

\author{
B. Prabhakaran \\ University of Texas at Dallas \\ P.O. Box 75083 \\ Richardson, Texas 75083 \\ praba@utdallas.edu
}

\begin{abstract}
Human gait has been studied in biomechanical and clinical research for a long time. Researchers use many different tools to detect and analyze human walking motions. 3D kinematic data captured by motion capture system is one of powerful tools to analyze the human motions. However, not many researches have focused on coordination of upper extremities during human locomotion. In this paper, we introduce the concept of feature extractions to analyze the upper and lower extremities on human gait. For analyzing coordination between arm joint and leg joint movement, we developed a gait visualization tool to measure velocity and angle of each arm joint and leg joint pair, and compare three different gait speeds (e.g., slow walk, casual walk, and fast walk) for each feature extraction.
\end{abstract}

\section{Keywords}

Upper Extremities, Lower Extremities, Gait Cycle, Motion Capture, ANOVA, Motion Data, Feature Extraction.

\section{INTRODUCTION}

Walking is the most common daily-functional activity among people. When people walk, swinging motion of their arms along with bipedal action is a natural human behavior. Positional and rotational movements of hand and leg joint occur in a coordinated fashion between upper and lower body segments. However, these actions may not be very well coordinated in disease states such as arthritis. This smoothness and coordination in human movement is reflected in the complexity involved in designing the walking motion in a humanoid robot. Therefore, it is important to understand the biomechanics and coordination of upper and lower extremity movements while walking. In particular, the rotational movements of hand and leg joints: a correlation between position of arm joints and leg joints (position angles of left arm and right leg and right arm and left leg) and a correlation between acceleration of rotational movements of arm joints and leg joints are important to study.

There are many different tools and techniques to analyze human gait [1][4][6][13][10]. 3D kinematic motion data captured by the $3 \mathrm{D}$ motion capture system is one of powerful tools to

Permission to make digital or hard copies of all or part of this work for personal or classroom use is granted without fee provided that copies are not made or distributed for profit or commercial advantage and that copies bear this notice and the full citation on the first page. To copy otherwise, or republish, to post on servers or to redistribute to lists, requires prior specific permission and/or a fee

BodyNets'09, April 1-3, 2004, LA, CA,USA

Copyright 2008 ICST 978-963-9799-41-7 ..\$5.00.

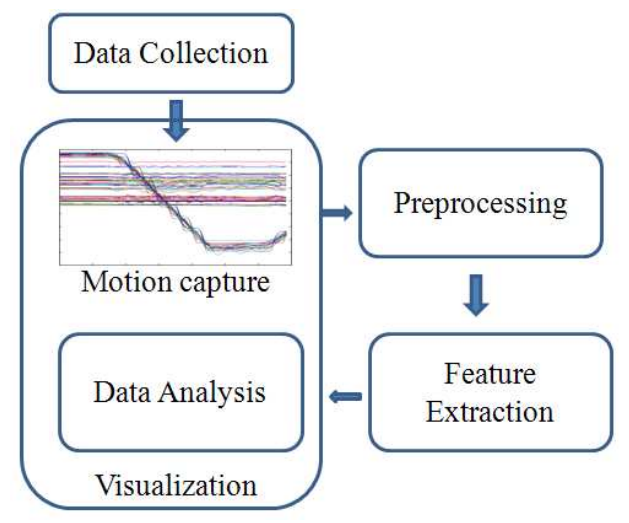

Figure 1. Motion data analysis flow

capture human motion. The advantages of 3D motion data are: 1) visualizing positional and angular changes of human joints without any extra visualization tool and 2) capturing speed acceleration of each body segments.

In this paper, we try to study the walking pattern of human locomotion and hand and leg coordination using 3D kinematic data. Our main objective is to understand and analyze walking that can provide the guiding information for a natural human walk. To find correlations between arms and legs, we chose the crosspairs of data in upper and lower body segments. In order to understand the natural human gait, obviously, we cannot ignore the balancing coordination of pendular movement of arms. For this, we introduce a gait cycle visualization tool and analyze the positional correlation of cross paired arm and leg kinematic -- left arm joint/right leg joint (LHRF), right arm joint/left leg joint (RHLF). Our data analysis indicated that there are 11 feature points (see chapter 3.5 for detail explanation on feature extractions) which can distinguish three walking speeds of natural human gait pattern. Figure 1 shows the flow of our approach to visualize and analyze upper and lower human body segments during the gait cycle.

\section{Related Study}

The long history of gait study started with the development of photograph at the early 1900 [7]. Many researchers studied normal gait as well as locomotion after certain disability factors such as stroke, Parkinsonism, Cerebellar disease and so on[1][4] $[8][15]$ 

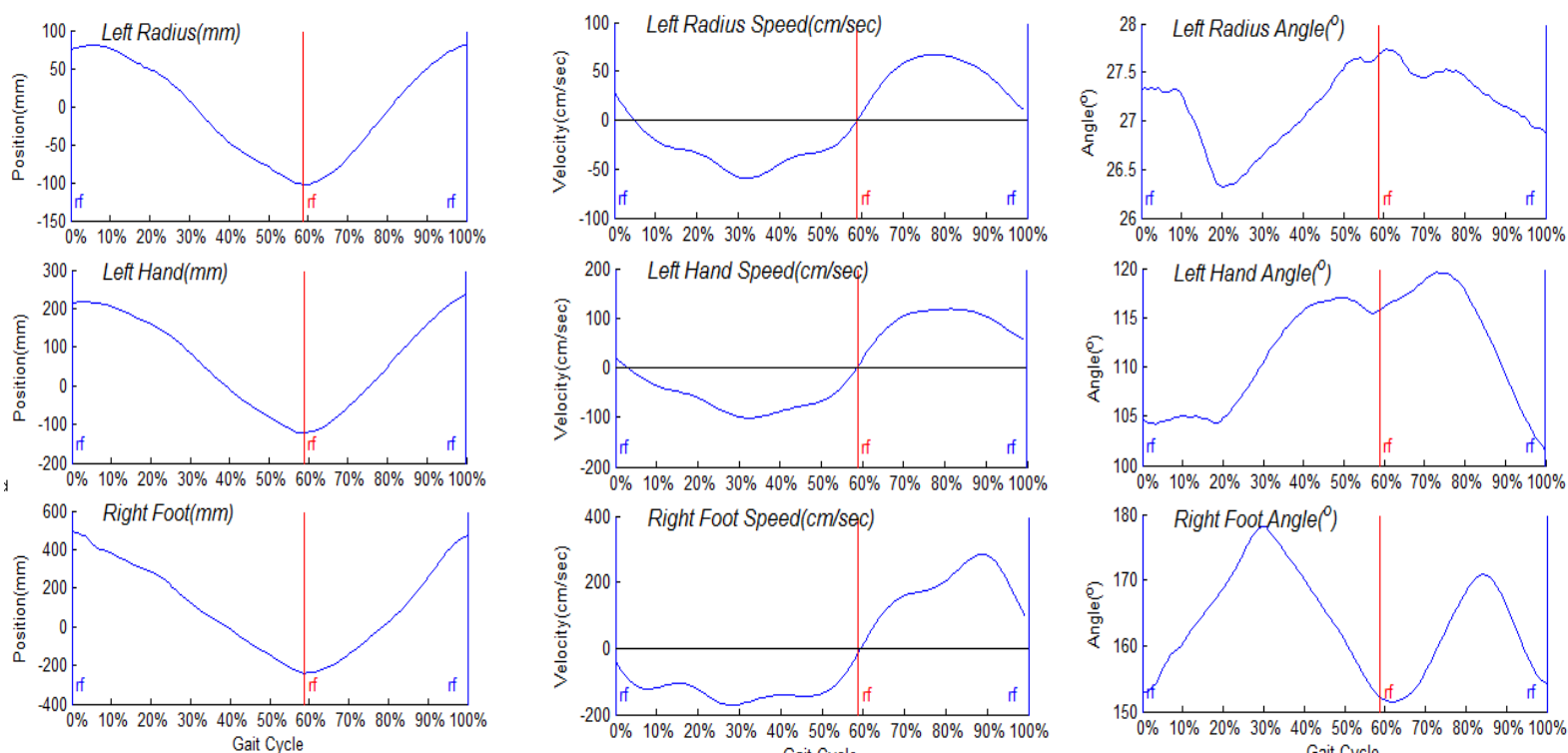

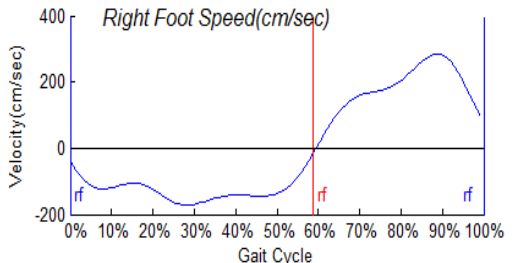

(b) Velocity time series

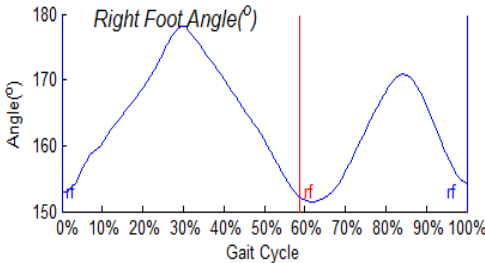

(c) Angular time series

Figure 2. Three visualized time series

\subsection{Gait Capturing Tool}

Many different tools have been used to capture features of human locomotion which cannot be seen with the naked eye. [8] introduced three measurement tools in Biomechanics: 3D motion capture systems (for measuring kinematics), force plates (for measuring kinetics), and Electromyography sensors (for measuring electrical muscle activity).

However, these tools have some limitations-cost effectiveness and probability. Recently some researchers were focused on more affordable solutions. An accelerometer is one of the tools to capture quantitative data because of its portability and relative accuracy [4][6][13].

Kito and Yoneda used a light weight distance meter to measure the speed and angle of participant to see whether the cycle duration is the dominant factor [10].

\subsection{Upper Extremities during Gait Cycle}

Compared to the long history of gait analysis, the study on upper extremities during gait has a relatively short history. The pendular action of hand swing during human locomotion has more degrees of freedom than gait thereby making it more complicated to analyze human locomotion [11]

P. Requejo et al. used compression-closing footswitches and 3D kinematic model with 7 rigid body segments on upper extremities and computed cadence, walking speed, stride length, stance duration, and each joint angle. For data analysis, P. Requejo et al. used thorax, shoulder, elbow, and wrist in the upper extremities to calculate sagittal plane joint motion (flexion/ extension) and captured the peak force and each joint angle [12]

B. Slavens et al. used 3D kinematic model with the upper arm, forearm, hand, upper crutch (UC), and lower crutch (LC), to measure crutch peak forces [13]. However, both [13] and [12] particularly focused on walking with crutches.
R. Emmerik and R. Wagenaar used an accelerometer to compute speed as well as angles of two groups of people (control group and Parkinson's disease group) walking on a computer controlled treadmill. For data analysis, they used four different sets of joint pairs: 1) left arm/right arm, 2) left leg/right leg, 3) left arm/left leg, and 4) right arm/right leg [4].

J. Stephenson et al. measured the self-selected speed of normal walking and fast walking speed between the healthy group and the stroke group. Then, they captured the relative phase (lag between the peek shoulder angle and the peek hip angle) and the frequency relation on hip and shoulder angle time series to assess the coordination between upper and lower extremities [14].

\section{Experiment Procedure}

In order to analyze the feature point, we made a visualization tool using Matlab. In our approach, instead of observing a fixed gait percent point, we chose several unique feature points for each participant. Since each walking trial with the same participant have differences in their gait cycles, it is more relevant to choose feature point dynamically rather than to choose a fixed gait cycle point (e.g., instead of choosing 65\% for phase change point, choose an actual phase change point in each individual).

\subsection{Participants}

We conducted walking data collection in ten healthy participants. Eight males and two females with ages of 23 to 53 years old performed walking motions in a controlled environment (Table 1). Each participant performed five trials at three different walking speeds. For the data accuracy, instead of walking on a treadmill, we asked each participant to walk at their self-selected three different walking velocities. 
Table 1. Participants' Physical characteristics

\begin{tabular}{|l|lllll|}
\hline & Median & Mean & STD & Min & Max \\
\hline Age (years) & 29 & 32.6 & 11.057 & 23 & 53 \\
Height $(\mathrm{cm})$ & 175 & 173.8 & 7.159 & 156 & 181 \\
Weight $(\mathrm{kg})$ & 75 & 73.72 & 11.01 & 50.8 & 89 \\
\hline
\end{tabular}

\subsection{Data collection}

For analyzing upper and lower extremities in human gait, we used 16 Vicon MX cameras to capture 3D kinematic data. The captured 3D motion capture data gives the positional and orientational information of 19 body segments at a frequency of $120 \mathrm{~Hz}$.

\subsection{Preprocessing}

Participants initially stood still and started walking by moving his/her foot forward. In our experiment, participant is walking along the $x$-axis. Figure 3 (a) illustrates the original data capture from the 3D motion capture system. Notice that once the participant's leg reaches its peak stride, it does not move, so the line curve remains flat. To obtain an accurate walking motion, the origin moves along $\mathrm{x}$-axis with human locomotion (Figure 3 (b)). Figure 3 (c) represents one gait cycle segment of a whole walking motion.

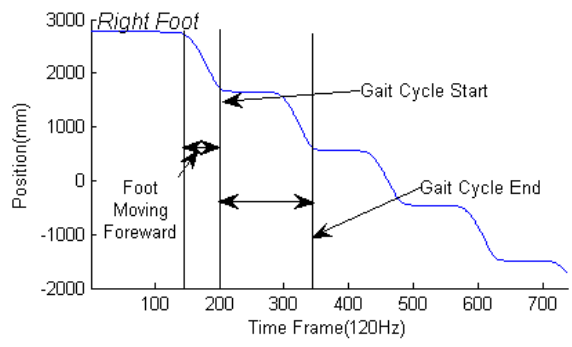

(a) Original Data

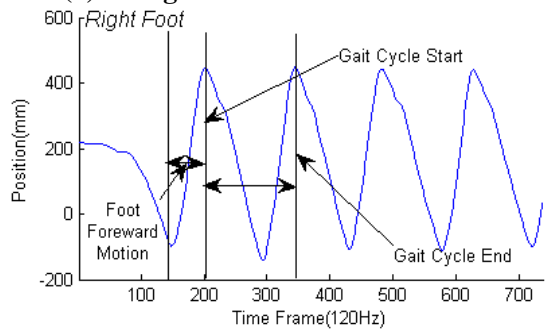

(b) Geometric conversion of data

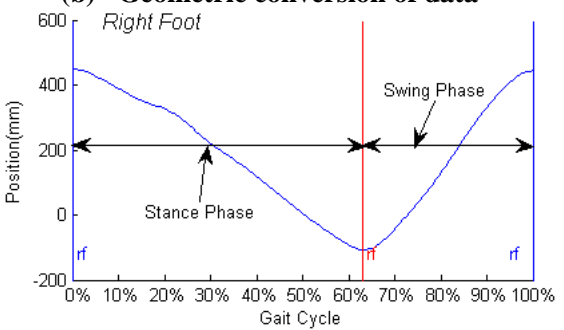

(c) Gait cycle of selected time interval

Figure 3. 3D motion capture time series data

\subsection{Upper and Lower Extremities Visualization Tool}

The most common observable features in gait analysis are joint positions, joint angles, and joint velocities [17][16]. We propose a visualization tool that can help to visualize these three different aspects with cross pair wise analysis: left lower body segments and right upper body segments; and vice versa. The positional time series (Figure 2. (a)) captures the stride length as well as a phase change point in the gait cycle from stance phase to swing phase. The velocity time series (Figure 2. (b)) captures speed variation occurring during the gait cycle in three body segments. Finally, the angular time series shows the angle changes within each segment's movements (Figure 2. (c)).

\subsection{Feature Extraction}

For observing and finding the correlation between leg joint movements and arm joint movements, we used cross pairs of upper extremities and lower extremities since one major function of the arm motion during walk is balancing. To compare three walking speeds (slow walk, normal walk, and fast walk), we carefully selected 24 feature points for each cross pair set. For cross each pair set the ipsilateral is the standard of the gait cycle. For example, RALF feature points are calculated based on the left leg gait cycle and vice versa. In each pair set, we chose the three body segments: a humerus segment, a hand segment, and a foot segment (Figure 4).

For feature extraction, we considered two selection points; 1) the phase change point from stance phase to swing phase that are varies in each participant and 2) velocity time series has more noticeable changes before and after a phase change. Every participant has different walking patterns and phase change moments. Even within the same participant during different walking trials, those moments are not similar sometimes. Hence we identified the following as our feature points: velocity time series' pick points, phases change point and zero velocity points in the upper extremities. Table 2 and Figure 5 indicate our feature points.

For measuring speed and angles of the leg and arm joint motions, we used time and distance differentiation (1) and vector angle function (2); and a 3rd order Butterworth filter with normalized cutoff frequency of $42 \mathrm{~Hz}$. We set the participant's waist as the origin of each axis; therefore for measuring angles of each arm and foot segment, we set a vector from an origin(waist) to a clavicle as a base vector and use arctan function to measure angles between $0^{\circ}$ to $180^{\circ}$ (See Figure 4 and Equation 2).

$$
\begin{aligned}
& \operatorname{Velocity}(t)=\frac{\Delta x}{\Delta t}, \text { where } \mathrm{t} \text { is time and } \mathrm{x} \text { is distance } \\
& \text { Angle }(t)=\arctan \left(\frac{|B(t) \times C(t)|}{B(t) \bullet C(t)}\right),
\end{aligned}
$$$$
\text { where } \mathrm{t} \text { is time and } \mathrm{B}(\mathrm{t}), \mathrm{C}(\mathrm{t}) \text { are joint vectors }
$$ 


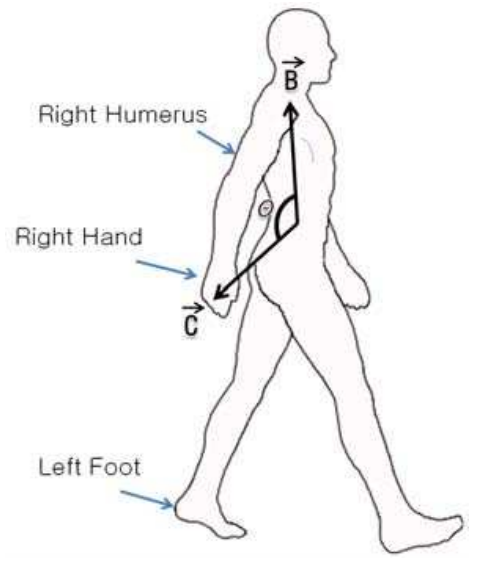

Figure 4.Obtained angle from equation (2)

Table 2. Feature points

\begin{tabular}{|ll|}
\hline \multicolumn{1}{|c|}{ Feature Description } \\
\hline F1 & First down peak speed(Humerus) \\
\hline F2 & F1 Angle(Humerus) \\
\hline F3 & Gait percent on Speed Zero point (Humerus) \\
\hline F4 & F3 angle (Humerus) \\
\hline F5 & Phase change point (Humerus) \\
\hline F6 & F5 angle (Humerus) \\
\hline F7 & Slope on phase change point(Humerus) \\
\hline F8 & First up peak speed(Humerus) \\
\hline F9 & F8 angle(Humerus) \\
\hline F10 & First down peak speed(Hand) \\
\hline F11 & F10 angle(Hand) \\
\hline F12 & Gait percent on Speed Zero point (Hand) \\
\hline F13 & F12 angle (Hand) \\
\hline F14 & Phase change point (Hand) \\
\hline F15 & F15 angle (Hand) \\
\hline F16 & Slope on phase change point(Hand) \\
\hline F17 & First up peak speed(Hand) \\
\hline F18 & F17 angle(Hand) \\
\hline F19 & First down peak speed(Foot) \\
\hline F20 & F19 angle(Foot) \\
\hline F21 & Last down peak speed(Foot) \\
\hline F22 & F21 angle(Foot) \\
\hline F23 & First up peak speed(Foot) \\
\hline F24 & F23 angle(Foot) \\
\hline & \\
\hline
\end{tabular}

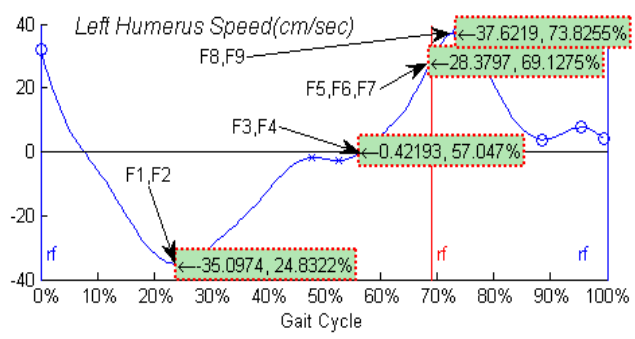

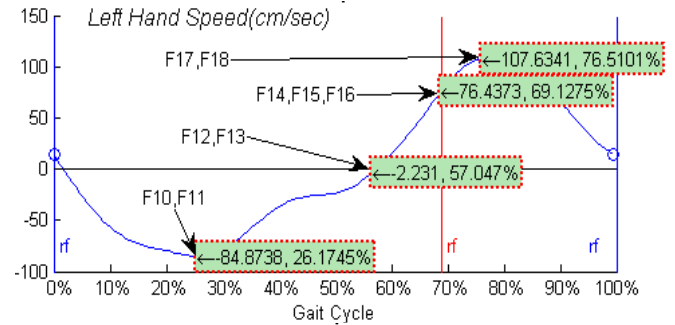

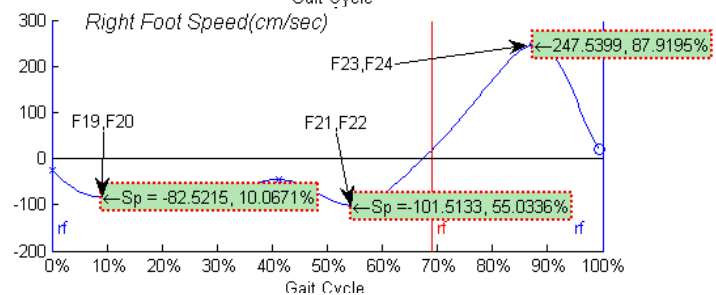

Figure 5. 24 Feature points on $\mathrm{X}$-axis of 3D motion capture time series (For fully implemented version of Tool refer Appendix A)

\subsection{Data Analysis}

For data analysis, we used the one way Analysis of Variance (ANOVA) to compare three different speeds of LHRF and RHLF. The ANOVA is one of statistical analysis techniques. One way ANOVA tests for the differences among two or more of independent groups. To see the difference among group, F-test and p-value are used. In general if the F-value increase the pvalue decrease. Equation 3-6 indicates the calculation of F-value.

$$
\begin{aligned}
& F=\frac{\left(\mathrm{SS}_{\text {among }} / \mathrm{a}-1\right)}{\left(S S_{\text {within }} / a(n-1)\right)} \\
& S S_{\text {within }}=\sum_{a} \sum_{n}(Y-M)^{2} \\
& S S_{\text {among }}=n \sum_{a}(M-G)^{2} \\
& G=\frac{1}{\text { an }} \sum_{a} \sum_{n} Y
\end{aligned}
$$

Where, $S S_{\text {among }}$ is the sum of squares among groups,

$S S_{\text {within }}$ denotes the sum of squares within groups $\mathrm{a}$ is the number of groups,

$\mathrm{n}$ is the number of observations within each group $\mathrm{Y}$ is individual observations within each group $M$ is a mean of each group $\mathrm{G}$ is grand mean.

For feature analysis, we set two different comparison aspects.

1) Comparison on three different speeds of LHRF and RHLF.

A. Dependent parameter: walk speed

B. Independent parameter: Each feature point 


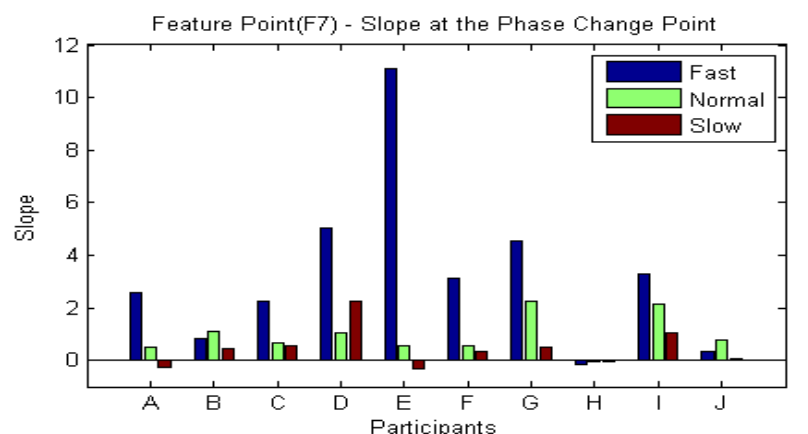

(a) Each participant's ('A'-'J') avg slope with three walking speeds.(F7)

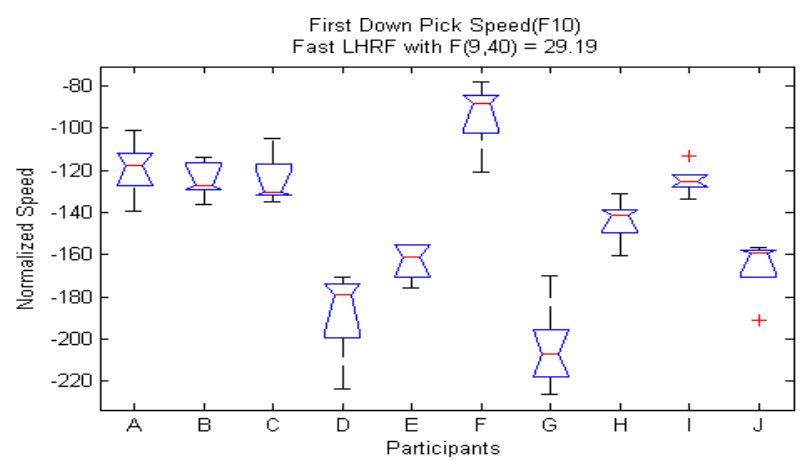

(c) Comparison between each participant's down pick speed (F10)

Figure 6. Participants' data analysis result of feature points

2) Comparison on among participants' each feature point of LHRF and those of RHLF.

A. Dependent parameter: Every participant

B. Independent parameter: Each feature point and walk speed.

Since each participant had a different stride lengths and walking speeds, we normalized each person's performance and compared the speed variations for comparison on each participant's performance.

\section{Result}

Based on the analysis of each person's LHRF and RHLF pair, we found that the feature points F2, F4, F6, F13, F15, F20, and F22 were significant with $\mathrm{p}<0.01$ during the fast walking trial; F1, F2, F4, F6 F11, F13, F15, F20, and F22 during the normal walking trial; and F1, F2, F4, F6, F9 F13 F15, and F22 during the slow walking trial were significant with $\mathrm{p}<0.01$ (Figure 6(d))

For comparisons between every participant's feature points, all feature points were significantly different with $\mathrm{p}<0.01$ in all walking speeds. This indicates that every participant has his/her unique gait pattern (Figure 6(a) and (c)). In fact there were significant differences from trial to trial within the same participant. (Figure 6(b)).

For comparison result among three different walking speeds, 10 feature points (F1, F7,F8, F10, F14, F16, F17, F19, F21, and F23)

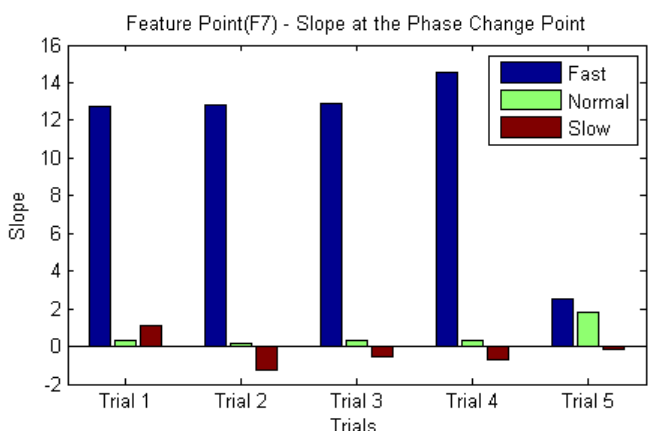

(b) Five different trials result of the same person's performance (F7)

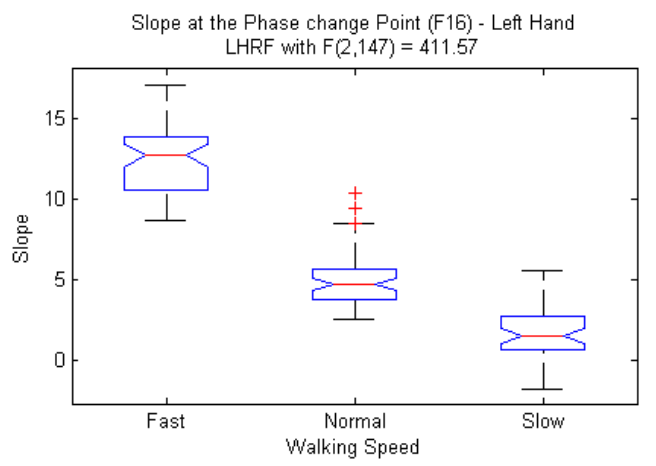

(d) Comparison between three walking speeds - Left hand slope at the phase change point (F16)

on LHRF; and 11 feature points (F1, F7, F10, F11, F16, F17, F19, F20, F21, F23, and F24) on RHLF were significant with $p<0.01$ (Table 3).

The most common area of significant feature points on the comparison among three walk speeds occurred at the F1, F7, F10, F16, F17, F19, F21, and F23. Based on this result, we may conclude that we can differentiate three different walking speeds in each participant at those feature points. For example, if one participant in experiment group has his/her slope at the phase change point of left hand speed is 13 (norm value), it may indicate that this person's walking speed is likely in the fast walk category (Figure 6(d)). Of course, we need to consider other significant feature points for better understanding of the overall performance.

Table 3. F and P-value of three different walk speed

\begin{tabular}{|l|r|r|r|r|}
\hline & \multicolumn{1}{|c|}{ LHRF } & & \multicolumn{1}{l|}{ RHLF } & \\
\hline Feature & F(2,147) & p-value & F(2,147) & p-value \\
\hline F1 & $\mathbf{2 5 . 7 9 0 2}$ & $\mathbf{2 . 5 1 E - 1 0}$ & $\mathbf{2 2 . 0 5 3 3}$ & $\mathbf{4 . 2 1 E - 0 9}$ \\
\hline F2 & 0.3667 & 0.6937 & 0.2137 & 0.8078 \\
\hline F3 & 6.0032 & 0.0031 & 3.0124 & 0.0522 \\
\hline F4 & 0.0023 & 0.9977 & 1.0869 & 0.34 \\
\hline F5 & 1.4189 & 0.2453 & 2.3886 & 0.0953 \\
\hline F6 & 1.3918 & 0.2519 & 0.9973 & 0.3714 \\
\hline F7 & $\mathbf{2 2 . 6 2 3}$ & $\mathbf{2 . 7 2 E - 0 9}$ & $\mathbf{2 0 . 4 4 1 8}$ & $\mathbf{1 . 4 7 E - 0 8}$ \\
\hline F8 & 8.0811 & $4.68 E-04$ & 0.5039 & 0.6052 \\
\hline F9 & 1.4836 & 0.2302 & 0.7005 & 0.498 \\
\hline
\end{tabular}




\begin{tabular}{|l|r|r|r|r|}
\hline F10 & $\mathbf{1 2 . 0 0 7 8}$ & $\mathbf{1 . 4 8 E - 0 5}$ & $\mathbf{1 3 . 9 9 4 3}$ & $\mathbf{2 . 7 3 E - 0 6}$ \\
\hline F11 & 1.4701 & 0.2333 & 11.0879 & $3.27 \mathrm{E}-05$ \\
\hline F12 & 1.0356 & 0.3576 & 5.2813 & 0.0061 \\
\hline F13 & 1.6026 & 0.2049 & 0.6966 & 0.4999 \\
\hline F14 & 5.7904 & 0.0038 & 0.6139 & 0.5426 \\
\hline F15 & 1.3918 & 0.2519 & 0.9973 & 0.3714 \\
\hline F16 & $\mathbf{1 6 7 . 9 6 9}$ & $\mathbf{0}$ & $\mathbf{1 3 5 . 0 1 2}$ & $\mathbf{0}$ \\
\hline F17 & $\mathbf{3 8 . 9 9 7 5}$ & $\mathbf{2 . 5 9 E - 1 4}$ & $\mathbf{2 9 . 6 0 1 1}$ & $\mathbf{1 . 5 7 E - 1 1}$ \\
\hline F18 & 2.8499 & 0.0611 & 0.7807 & 0.46 \\
\hline F19 & $\mathbf{7 5 . 3 3 8 9}$ & $\mathbf{0}$ & $\mathbf{8 2 . 5 7 8 5}$ & $\mathbf{0}$ \\
\hline F20 & 3.4983 & 0.0328 & 7.5738 & $7.40 \mathrm{E}-04$ \\
\hline F21 & $\mathbf{1 4 1 . 5 9}$ & $\mathbf{0}$ & $\mathbf{1 8 3 . 5 6 8}$ & $\mathbf{0}$ \\
\hline F22 & 0.0333 & 0.9672 & 1.8547 & 0.1601 \\
\hline F23 & $\mathbf{8 4 . 2 7 8 8}$ & $\mathbf{0}$ & $\mathbf{7 3 . 7 2 9 3}$ & $\mathbf{0}$ \\
\hline F24 & 3.0466 & 0.0505 & 5.3545 & 0.0057 \\
\hline
\end{tabular}

\section{Conclusion}

In this paper, we introduce $d$ the concept of cross pair wise gait analysis of upper and lower extremities as well as the visualization tool for human locomotion. The objective of our approach is to find features that can separate distinctive walking speeds and find the normal walking pattern. For this we introduced the upper and lower extremities visualization tool which visualizes extracted features of the upper body segments during human gait to understand any correlation between upper extremities and lower extremities.

Compared to the limited degree of freedom of gait, arms have more freedom which makes the study of upper extremities more difficult. 3D kinematic data is as powerful as any other gait capturing system, yet it only provides the visible movement analysis of human gait. To better understand natural walking motion, it is necessary to integrate external data (e.g., 3D kinematic data) with internal force data (e.g., kinetic data).

\section{REFERENCES}

[1] T. Annaswamy, C.Giddings, U Croce, and C Kerrigan, "Rectus Femoris: Its Role in Normal Gait," Archives of Physical Medicine \& Rehabilitation, 80(8):930-934, 1999

[2] B. Bilney, M. Morris and K. Webster, "Concurrent related validity of the GAITRite walkway system for quantification of the spatial and temporal parameters of gait," Gait and Posture, 17(1):68-74, February 2003.

[3] M. Dutton, Orthopaedic: Examination, Evaluation, and Intervention, New-York: MacGraw-Hill, 2008.

[4] R. Emmerik and R. Wagenaar, "Dynamics of movement coordination and tremor during gait in Parkinson's disease," Human Movement Science, 15(2):203-235, 1996
[5] D. Farris, G. Urquizo, D. Beattie, T. Woods, and D. Berghaus, "A Simplified Accelerometer System for Analysis of Human Gait," Experimental Techniques, 17(1):33 - 36, Jan 2008.

[6] D. Gafurov, K. Helkala, and T. Sondrol, "Biometric Gait Authentication Using Accelerometer Sensor," Journal of Computers, 1(7):51-59, Nov. 2006.

[7] M. Kadaba, H. Ramakrishnan, and M. Wootten, "Measurement of lower extremity kinematics during level walking." Journal of Orthopedic Research. 8(3):383-92, May 1990.

[8] D. Kerrigan and T Annaswamy, "Biomechanical Correlates of Movement: Principles of Gait," Exercise in Rehabilitation Medicine, 1999, ed. Frontera W \& Slovik D, Human Kinetics, Chicago, IL.

[9] D. Kerrigan and T Annaswamy, "The Functional Significance of Spasticty as Assessed by Gait Analysis," Journal of Head Trauma Rehabilitation, 12(6):29-39,1997

[10] T. Kito and T. Yoneda, " Dominance of gait duration in casual walking," Human Movement Science, 25:383-392, 2006

[11] G. Rau, C. Disselhorst-Klug, and R. Schmidt, "Movement biomechanics goes upwards: from the leg to the arm," Journal of Biomechanics, 33(10):1207-1216, October 2000.

[12] P. Requejo, D. Wahl, E. Bontrager, C. Newsam, J. Gronley, S. Mulroy, and J. Perry, "Upper extremity kinetics during Lofstrand crutch-assisted gait," Medical Engineering \& Physics, 27(1):19-29, 2005.

[13] B. Slavens, J. Frantz, P. Sturm, and G. Harris, "Upper Extremity Dynamics During Lofstrand Crutch-Assisted Gait in Children With Myelomeningocele," Journal of Spinal Cord Medicine, 30(1): S165-S171,2007.

[14] J. Stephenson, A. Lamontagne, and S. De Serres, "The coordination of upper and lower limb movements during gait in healthy and stroke individuals," Gait \& Posture, 29(1):11$16,2009$.

[15] M. Thirunarayan, D. Kerrigan, M Rabuffetti, U. Croce, and M. Saini, "Comparison of three methods for estimating vertical displacement of center of mass during level walking in patients," Gait \& Posture, 4:306-314, 1996

[16] X. Wang, Z. Yu, H. Wong, "Searching of Motion Database based on Hierarchical SOM," IEEE International conference on Multimedia and Expo 2008, pp.210-220, Dec. 2007

[17] J. Xiang, J. Weng, Y. Zhuang, and F. Wu, "Ensemble Learning HMM for Motion Recognition and Retreval by Isomap Demention Reduction," Journal of Zhejing University Science, 12(7A):2063-2072, 2006 


\section{Appendix A}

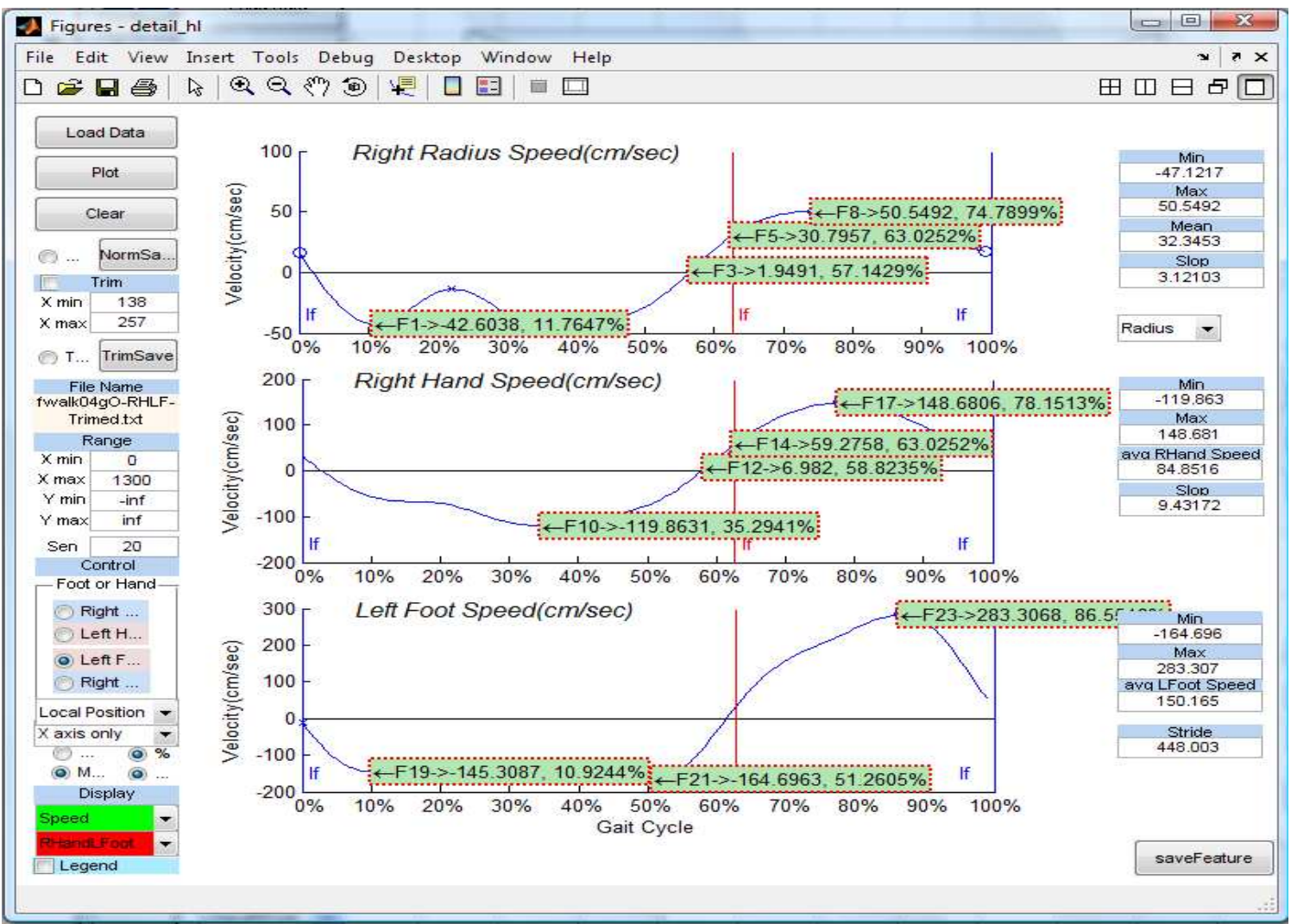

Figure 7. Gait Cycle Visualization Tool 